\title{
Review of: "miR1908-5p regulates energy homeostasis in hepatocyte models"
}

Young Hee $\mathrm{Choi}^{1}$

1 Dongguk University

Potential competing interests: The author(s) declared that no potential competing interests exist.

This paper identified the function of miR-1908-5p to regulate key metaoblic regulators such as AKT, AMPK and LKB etc. in metabolic and energy regulation in hepatocyte models. The experimetal methods were well designed and the results sufficiently supported the hypothersis. 